\title{
Diagnostic otoscopy skills of community ear assistants in Western Nepal
}

\author{
R YOUNGS, N WEIR, P THARU, R B BOHARA, D BAHADUR BC \\ Britain Nepal Otology Service, Guildford, UK
}

\begin{abstract}
Introduction: Deafness is a major problem in developing countries. Rural communities tend to be affected more than urban ones, and chronic otitis media is common. The World Health Organization has proposed primary ear care as a method of providing otological services in developing countries. This study aimed to assess the diagnostic otoscopy skills of community ear assistants in rural Western Nepal.

Materials and methods: Community ear assistants undertook the pre-operative evaluation of 92 patients selected for middle-ear surgery in an 'ear camp' setting. The otoscopy skills of community ear assistants were also assessed by means of an otoscopy quiz. Consultant otologists and trainee otolaryngologists underwent an identical assessment.

Results: The community ear assistants' selection of patients for middle-ear surgery concurred with the consultant otologists' opinion in 87 of 92 patients (94.5 per cent). The level of community ear assistants' otoscopy skills was between that of junior and senior otolaryngology trainees.

Conclusions: With intensive training, medically unqualified community ear assistants can develop otoscopy skills comparable to those of medically qualified otolaryngology trainees. These results support the development of primary ear care in poorer countries where access to specialist otological services is difficult or impossible.
\end{abstract}

Key words: Deafness; Primary Health Care; Developing Countries; Otoscopy; Nepal

\section{Introduction}

The World Health Organization (WHO) estimates that 278 million people worldwide suffer from disabling deafness. ${ }^{1}$ The majority of people with hearing disability live in developing countries. The implications of significant hearing loss are severe. In childhood, adequate hearing levels are required for the development of normal speech and language. In adulthood, those with hearing loss have difficulty finding employment. In older persons, deafness is associated with social isolation.

Countries in low and middle income groups, as defined by the World Bank, are termed developing countries. $^{2}$ These countries do not have the resources to develop highly specialised diagnostic and treatment facilities for those with hearing impairment. It is understood that the strategies used to effectively improve care for deaf people in developing countries will differ from those used to manage the same diseases in rich countries. At a basic level, the importance of education regarding the causes and implications of hearing loss has been emphasised. ${ }^{3}$ This approach focuses on the need for good nutrition, appropriate immunisations, and the avoidance of noise and ototoxic medications.
In rich countries, treatment of ear disease is often carried out by specialist otologists who have higher medical qualifications. However, access to specialised otological services is generally not available for those in developing countries, a situation worsened by the fact than the prevalence of hearing loss is higher in rural than urban areas. ${ }^{4}$ In acknowledgement of this situation, the WHO has recommended the development of so-called 'primary ear care'. 5 In this approach, basic prevention, diagnosis and treatment of ear disease is undertaken by suitably trained primary ear care workers, who are not medically qualified.

In Western Nepal, the Britain Nepal Otology Service, in association with the Nepal Red Cross, has been delivering a community-based ear care programme for more than 10 years. ${ }^{6}$ This programme is based on the employment of community ear assistants: workers with basic technical qualifications who receive intensive training in the prevention, diagnosis and treatment of ear disease. The service provided by these community ear assistants is augmented by twiceyearly visiting surgical teams composed of British and Nepali otologists, nurses and audiologists who undertake intensive 'ear camps'. 
During the course of a typical Britain Nepal Otology Service ear surgery camp, approximately 100 patients will undergo major middle-ear surgery (i.e. myringoplasty, mastoidectomy and stapedotomy) over a two week period. Published reports from other such programmes shows this to be an effective method of delivering good quality tertiary level ear care to rural populations. ${ }^{7}$ The selection of patients for otological procedures in Britain Nepal Otology Service ear camps, and their post-operative follow up, is now undertaken by community ear assistants. Hence, it is important that these community ear assistants possess adequate skills in diagnostic otoscopy.

Formal assessment of diagnostic otoscopy skills has received little attention in the otological literature. Wormald and colleagues have described a structured teaching method for use with otology trainees, which uses photographs of ears taken with a Hopkins rod endoscope. ${ }^{8}$

The aim of the present study was to formally evaluate the otoscopy skills of community ear assistants in Western Nepal.

\section{Materials and methods}

Assessment of the otoscopy skills of community ear assistants was undertaken during the 38th Britain Nepal Otology Service surgical ear camp. This ear camp took place from 22 March to 1 April 2009 at The Fateh-Bal Eye Hospital in Nepalgunj, Nepal. Otoscopy skills were assessed by two different methods.

Ninety-two potential surgical patients had previously been selected by three community ear assistants. Before surgery was undertaken, each patient was examined by a consultant otologist from the visiting team. Both ears were examined, giving 184 otoscopy events for analysis. The observer disagreement between community ear assistants and consultant otologists was analysed using kappa statistics. At the time the community ear assistants had selected the surgical patients, prior to the ear camp, they had been unaware that the quality of their assessment would be subject to formal evaluation.

The second method of assessment involved testing the recognition of otoscopic pathology by inspection of 50 consecutive otoscopic images on a computer screen. Test scoring was dependent on recognition of the following features: side of ear, diagnosis, presence of cholesteatoma, presence of perforated tympanic membrane and any other notable features. One minute was allowed for each otoscopic image. A maximum of 10 marks were allocated for each image, giving a maximum score of 500 marks. The same assessment of otoscopy skills was also administered to the three consultant otologists attending the camp. Subsequently in the UK (at Gloucestershire Royal Hospital), the assessment was administered to four junior medical trainees (at Foundation Year Two (i.e. senior house officer) level), three senior trainees (at

\begin{tabular}{|c|c|c|c|}
\hline OTOSCOP & $\begin{array}{l}\text { T } \\
\text { KILLS OF C } \\
\text { D CONSUL }\end{array}$ & $\begin{array}{l}\text { 3LE I } \\
\text { MMUNITY } \\
\text { NT OTOLO }\end{array}$ & $\begin{array}{l}\text { AR ASSISTANTS } \\
\text { ISTS* }\end{array}$ \\
\hline CO Dx & CEA & $\mathrm{x}(n)$ & Total ears $(n(\%))$ \\
\hline & Diseased ear & Normal ear & \\
\hline Diseased ear & 128 & 0 & $128(69.6)$ \\
\hline Normal ear & 6 & 50 & $56(30.4)$ \\
\hline $\begin{array}{r}\text { Total ears } \\
(n(\%))\end{array}$ & $134(72.8)$ & $50(27.2)$ & $184(100)$ \\
\hline
\end{tabular}

*For 184 ears assessed during 38th Britain Nepal Otology Service ear camp. $\mathrm{CO}=$ consultant otologist; $\mathrm{Dx}=$ diagnosis; $\mathrm{CEA}=$ community ear assistant

specialist registrar level) and two specialist doctors (a staff grade doctor and an associate specialist).

\section{Results}

\section{Assessment of ear camp surgical patients}

The community ear assistants had selected 92 patients for surgery. Of these, the visiting consultant otologists agreed with the community ear assistants' otoscopic assessment and surgical decision in 87 patients $(94.5$ per cent). Of the 87 patients selected for surgery, 62 were selected for repair of perforated tympanic membranes (myringoplasty), 18 for eradication of cholesteatoma (mastoidectomy) and seven for otosclerosis surgery (stapedotomy). The contralateral ears were also examined. Using the presence or absence of

\begin{tabular}{lcccc}
\multicolumn{5}{c}{ TABLE II } \\
OTOSCOPY QUIZ SCORES \\
\hline Cdt no & CEA & JMT & SMT /AS & CO \\
\hline 1 & 322 & 320 & 363 & 421 \\
2 & 309 & 296 & 340 & 380 \\
3 & 292 & 294 & 335 & 358 \\
4 & & 269 & 330 & \\
5 & 308 & 295 & 329 & 386 \\
Total (mean) & 308 & 339 & \\
\hline
\end{tabular}

Cdt no $=$ candidate number; $\mathrm{CEA}=$ community ear assistant; JMT $=$ junior medical trainee (Foundation Year Two); SMT/ $\mathrm{OS}=$ senior medical trainee or associate specialist; $\mathrm{CO}=$ consultant otologist

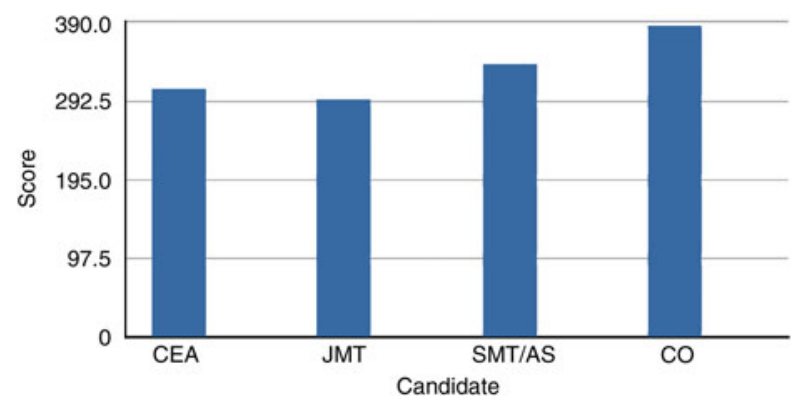

FIG. 1

Mean otoscopy quiz scores (maximum score 500). CEA = community ear assistant; JMT = junior medical trainee; SMT / AS = senior medical trainee or associate specialist; $\mathrm{CO}=$ consultant otologist 
otoscopic abnormality in 184 ears, a kappa coefficient of 0.93 was derived (where 1.0 would indicate perfect agreement between the community ear assistants and consultant otologists) (Table I). The community ear assistants had a slight tendency to over-diagnose otoscopic pathology. There were no cases in which the community ear assistants had failed to diagnose significant otological pathology.

- Deafness is a major public health issue in developing countries; rural communities are affected more than urban ones, and chronic otitis media is common

- The World Health Organization has proposed 'primary ear care' as a means of providing otological care in poorer countries

- This study evaluated the otoscopy skills of community ear assistants in Western Nepal

- Given adequate training, community ear assistants can develop good otoscopy skills; partnership between such workers and visiting surgical teams thus enables validated selection and follow up of surgical cases

\section{Otoscopy quiz}

The otoscopy quiz scores are shown in Table II, and represented graphically in Figure 1. The scores of the medically qualified individuals were consistent with seniority and length of training. The community ear assistants scored at a level between that of junior and senior otolaryngology trainees.

\section{Discussion}

This study suggests that community ear assistants in rural Nepal have developed good otoscopy skills. These skills have enabled community ear assistants to accurately assess patients with chronic otitis media and otosclerosis for potential major middle-ear surgery. When assessing patients for surgery, the community ear assistants tended to slightly over-diagnose ear disease in comparison with consultant otologists. On no occasion did the community ear assistants fail to diagnose significant otological pathology. Formal assessment with an otoscopy quiz graded the community ear assistants' otoscopy skills at between junior and senior otolaryngology trainee level. It is possible that this method of assessment favours medically qualified individuals, who may be more used to this assessment style. Also, the fact that the community ear assistants did not have English as their first language should be taken into account. It is hence possible that this study's assessment method underestimated the otoscopy skills of community ear assistants.

The availability of community ear assistants with good otoscopy skills is important when developing primary ear care as a method of managing ear disease in a developing world setting. When used in parallel with a visiting surgical team, the availability of such skilled primary health workers provides the opportunity for validated post-operative follow up. The results of this study would seem to vindicate the WHO policy of expanding primary ear care.

\section{References}

1 Deafness and hearing impairment. In: http://www.who.int/mediacentre/factsheets/fs300/en/ [8 February 2010]

2 World Bank. World Development Report 2004. Equity and Development. Washington DC: World Bank, 2006

3 Tucci DL, Merson MH, Wilson BS. A summary of the literature on global hearing impairment: current status and priorities for action. Otol Neurotol 2009;31:31-41

4 Kumar S. Deafness and its prevention - Indian scenario. Indian J Paediatr 1997;64:801-9

5 World Health Organization. Primary Ear and Hearing Care Training Resource, Advanced Level. Geneva: WHO Press, 2006; 11-12

6 Shrestha R, Baral K, Weir N. Community ear care delivery by community ear assistants and volunteers: a pilot programme. J Laryngol Otol 2001;115:869-73

7 Snidvongs K, Vatanasapt P, Thanaviratananich S, Pothaporn M, Sannikorn P, Supivaphun P. Outcome of mobile ear surgery units in Thailand. J Laryngol Otol 2009;24:382-386

8 Wormald PJ, Browning GG, Robinson K. Is otoscopy reliable ? A structured teaching method to improve otoscopic accuracy in trainees. Clin Otol 1995;20:63-7

Address for correspondence:

Mr Robin Youngs,

Consultant Otologist,

Gloucestershire Royal Hospital,

Great Western Road,

Gloucester GL1 3NN, UK

E-mail: robin.youngs@glos.nhs.uk

Mr R Youngs takes responsibility for the integrity of the content of the paper

Competing interests: None declared 